\title{
A Multimodal Approach to Understanding Human Vocal Expressions and Beyond
}

\author{
Shrikanth (Shri) Narayanan \\ University of Southern California \\ Los Angeles, CA \\ shri@sipi.usc.edu
}

\begin{abstract}
Human verbal and nonverbal expressions carry crucial information not only about intent but also emotions, individual identity, and the state of health and wellbeing. From a basic science perspective, understanding how such rich information is encoded in these signals can illuminate underlying production mechanisms including the variability therein, within and across individuals. From a technology perspective, finding ways for automatically processing and decoding this complex information continues to be of interest across a variety of applications.

The convergence of sensing, communication and computing technologies is allowing access to data, in diverse forms and modalities, in ways that were unimaginable even a few years ago. These include data that afford the multimodal analysis and interpretation of the generation of human expressions.

The first part of the talk will highlight advances that allow us to perform investigations on the dynamics of vocal production using real-time imaging and audio modeling to offer insights about how we produce speech and song with the vocal instrument. The second part of the talk will focus on the production of vocal expressions in conjunction with other signals from the face and body especially in encoding affect. The talk will draw data from various domains notably in health to illustrate some of the applications.
\end{abstract}

\section{Author Keywords}

Human signals; speech; seeing speech; real time MRI; individual variability; biometrics; affective computing; behavioral informatics

\section{BIOGRAPHY}

Shrikanth (Shri) Narayanan is the Niki \& C. L. Max Nikias Chair in Engineering at the University of Southern California, where he is Professor of Electrical Engineering, and jointly in Computer Science, Linguistics, Psychology, Neuroscience and Pediatrics, Director of the Ming Hsieh Institute and Research Director of the Information Sciences Institute. Prior to USC he was with AT\&T Bell Labs and AT\&T Research. His research focuses on humancentered information processing and communication technologies. He is a Fellow of the Acoustical Society of America, IEEE, ISCA, the American Association for the Advancement of Science (AAAS), Association for Psychological Science (APS) and the National

Permission to make digital or hard copies of part or all of this work for personal or classroom use is granted without fee provided that copies are not made or distributed for profit or commercial advantage and that copies bear this notice and the full citation on the first page. Copyrights for third-party components of this work must be honored. For all other uses, contact the Owner/Author.

ICMI'18, October 16-20, 2018, Boulder, CO, USA.

(C) 2018 Copyright is held by the owner/author(s)

ACM ISBN 978-1-4503-5692-3/18/10.

DOI: https://doi.org/10.1145/3242969.3243391
Academy of Inventors. Shri Narayanan is Editor in Chief for IEEE Journal of Selected Topics in Signal Processing and an Editor for the Computer, Speech and Language Journal and an Associate Editor for the APISPA Transactions on Signal and Information Processing. He is a recipient of several honors including the 2015 Engineers Council's Distinguished Educator Award, a Mellon award for mentoring excellence, the 2005 and 2009 Best Journal Paper awards from the IEEE Signal Processing Society and serving as its Distinguished Lecturer for 2010-11, as an ISCA Distinguished Lecturer for 2015-16 and the 2017 Willard R. Zemlin Memorial Lecturer for American Speech and Hearing Association. With his students, he has received several best paper awards including a 2014 Ten-year Technical Impact Award from ACM ICMI and a sixtime winner of the Interspeech Challenges. He has published over 750 papers and has been granted 17 U.S. patents.

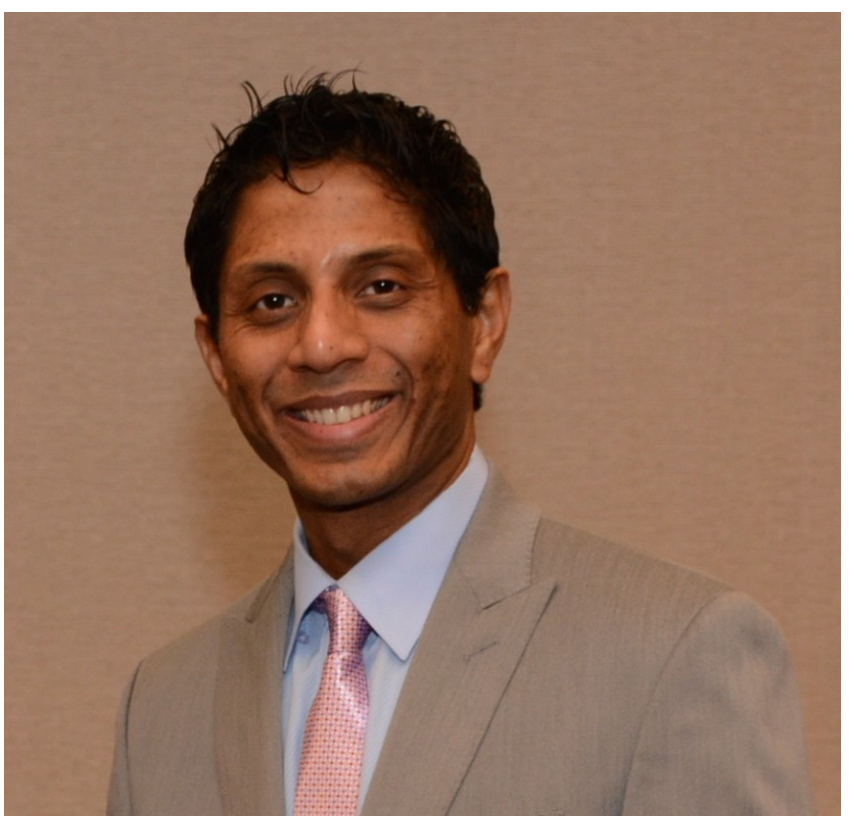

\section{REFERENCES}

1. Signal Analysis and Interpretation Laboratory https://sail.usc.edu/

2. Speech Production and Articulation Knowledge Group https://sail.usc.edu/span/

3. Daniel Bone, Chi-Chun Lee, Theodora Chaspari, James Gibson, and Shrikanth Narayanan. Signal Processing and Machine Learning for Mental Health Research and Clinical Applications. IEEE Signal Processing Magazine, 34(5):189-196, September 2017.

4. Shrikanth S. Narayanan and Panayiotis Georgiou. Behavioral Signal Processing: Deriving Human Behavioral Informatics from Speech and Language. Proceedings of IEEE, 101(5):1203 - 1233, may 2013. 